\title{
Correction to: Formalizing Natural Languages with NooJ 2018 and Its Natural Language Processing Applications
}

Ignazio Mauro Mirto, Mario Monteleone, and Max Silberztein

\section{Correction to:}

I. Mauro Mirto et al. (Eds.):

Formalizing Natural Languages with NooJ 2018 and Its Natural Language Processing Applications, CCIS 987, https://doi.org/10.1007/978-3-030-10868-7

In the original version of the book, The given name and family name of the first editor was tagged incorrectly. It has been corrected. 